\title{
WERNER SCHOLZE-STUBENRECHT \\ Duden 11 - Lexikografisches Konzept und lexikografische Praxis
}

\begin{abstract}
Die folgende Darstellung versucht, zur Konzeption und Praxis eines lexikografischen Projekts vor allem solche Informationen zu bieten, die nicht im Buch selbst nachzulesen sind oder von Kennern der Materie aus dem Buch herausgelesen werden könnten.

Am Anfang steht der Versuch einer Rekonstruktion der Planung und Erarbeitung der ersten Auflage des Duden 11 (Abschnitte 1 bis 6), dann folgt eine entsprechende Beschreibung für die zweite Auflage (Abschnitte 7 und 8) und abschließend ein kurzer Ausblick auf die aus heutiger redaktioneller Sicht mögliche Weiterentwicklung des Buches in einer dritten Auflage (Abschnitte 9 bis 11).

Der Schwerpunkt der Darstellung liegt auf den generellen Rahmenbedingungen und allgemeinen konzeptionellen Zielen und deren Auswirkungen auf Inhalt und Struktur dieses Wörterbuchs.
\end{abstract}

\section{Allgemeines zur Konzeption der ersten Auflage 1992}

Das Konzept für ein idiomatisches Wörterbuch der deutschen Gegenwartssprache innerhalb der Duden-Standardreihe stammt von Günther Drosdowski, der von 1973 bis 1995 Leiter der Dudenredaktion war. Er schrieb selbst, etwa von 1970 an, das Manuskript - zufälligerweise (vielleicht auch nicht ganz zufälligerweise) genau bis zum Stichwort Konzept mit Wendungen wie jmdn. aus dem Konzept bringen oder jmdm. nicht ins Konzept passen. Für weitere Arbeiten ließen ihm seine Aufgaben als Redaktionsleiter keine Zeit mehr, und so wurde die zweite Hälfte im Jahr 1976 von mir übernommen. Nach dem Gesetz der alphabetischen Reihe war das erste Stichwort, das mir zur Bearbeitung zufiel, genau das auf dem Plakat der IDS-Tagung 2003 abgebildete, nämlich Kopf. Zu den ersten Wendungen, mit denen ich mich auseinander setzte, gehörten nicht mehr wissen, wo einem der Kopf steht und für etw. seinen Kopf hinhalten müssen.

Mit der ersten Entscheidung, das Buch in die damals noch aus zehn Bänden bestehende Dudenreihe einzugliedern, waren Format und Umfang zumindest im Groben festgelegt: das mittelgroße Dudenformat von 12,5 x 19 $\mathrm{cm}$ und eine Gesamtseitenzahl von etwa 800. (Letztlich sind es 860 Seiten geworden, in der zweiten Auflage dann sogar 955.) Auch die konzeptionel- 
le Grundausrichtung war durch den allgemeinen Charakter der Reihe in gewisser Weise vorgegeben; die in diesem Rahmen zu bewältigenden Inhalte wurden von den folgenden Parametern bestimmt:

- Das Wörterbuch soll sich an ein möglichst breites Publikum wenden,

- es soll sich von den vorhandenen Konkurrenzwerken unterscheiden,

- es soll dem Stand der lexikografischen Entwicklung und der linguistischen Forschung Rechnung tragen

- und es soll auf den hauseigenen Quellen basieren oder zumindest durch diese abgesichert sein.

\section{Der Markt und die Zielgruppen}

Der Markt für idiomatische Wörterbücher wurde in den Sechzigerjahren im deutschsprachigen Raum im Wesentlichen von Publikationen der folgenden beiden Typen bedient: Zum einen gab es mit Wolf Friederichs Moderner deutscher Idiomatik (1966) eine - nach eigenem Bekunden ,sachlich-objektive" - Sammlung idiomatischer Redewendungen mit Definitionen und Anwendungsbeispielen. Zum Zweiten hatten sich zuvor schon verschiedene Werke auf dem Buchmarkt etabliert, die (wie zum Beispiel Kurt Krüger-Lorenzen mit seinem Das geht auf keine Kuhhaut [1960]) bekannte Redewendungen eher anekdotisch unter sprachgeschichtlichem Aspekt im Stil von Zeitungsglossen behandelten. Damit waren zwei Zielgruppen angesprochen: Von Friederich diejenigen, die sich über den korrekten Gebrauch von Redewendungen informieren wollten, also nicht zuletzt die Deutsch Lernenden, und von Krüger-Lorenzen die Gruppe, die wir gern ziemlich unscharf als die „sprachlich interessierten Laien“" bezeichnen, deren Interesse erfahrungsgemäß besonders gut mit sprach- und kulturgeschichtlichen Darlegungen geweckt werden kann.

Es lag für die Dudenredaktion nahe, mit einem eigenen Werk nach Möglichkeit beiden Benutzergruppen eine Alternative zu den vorhandenen Büchern zu bieten.

\section{Die zur Verfügung stehenden Quellen}

Die Sammlung und Auswahl der Einträge für ein idiomatisches Wörterbuch konnte sich zunächst auf schon recht umfassende Vorarbeiten in einem älteren Dudenband stützen: Bereits in der ersten Auflage des Duden-Stilwörterbuchs aus dem Jahr 1934 sind neben den freien Kollokationen der Stichwörter auch "Verbindungen, Redensarten, Wendungen" verzeichnet, allerdings im Text nicht deutlich hervorgehoben. In drei parallel zum Duden 11 entstandenen und deutlich früher als dieser abgeschlossenen Werken, in der ersten Auflage des Duden-Bedeutungswörterbuchs (1970), der sechsten Auflage des eben genannten Stilwörterbuchs (1971) und schließlich auch im 1981 abgeschlossenen Großen Wörterbuch der deutschen Sprache erhielten die festen Wendungen eine klare optische Unterscheidung von freien Kolloka- 
tionen durch halbfette Schrift - für diese Werke musste also von der Dudenredaktion erstmals eine explizite Differenzierung zwischen Kollokation und fester Verbindung durchgeführt werden. Dazu wurden die Probleme der Abgrenzung und der lexikografischen Behandlung der idiomatischen Wendungen mehrfach in Redaktionsbesprechungen, vor allem zum Großen Wörterbuch, diskutiert.

Das Verzeichnis der zum Thema herangezogenen Primär- und Sekundärliteratur im Einleitungsteil des Duden 11 zeigt, dass wir bei unseren Entscheidungsfindungen versucht haben, sowohl den Stand der linguistischen Forschung zu berücksichtigen als auch die Ergebnisse der praktischen Kodifizierung der deutschen Idiomatik in den verschiedensten Publikationen.

Eine verglichen mit den heutigen elektronischen Mitteln in Bezug auf den Umfang und auf die Zugriffsmöglichkeiten zwar begrenzte, für die damalige Zeit aber ausgesprochen hilfreiche Grundlage für die Entwicklung einer eigenständigen Wörterbuchsubstanz war die Duden-Sprachkartei, deren stichwortbezogene Dokumentation des schriftlichen Sprachgebrauchs der deutschen Gegenwartssprache in einer damals schon siebenstelligen Zahl von Belegen auch heute noch einige lexikografische Vorteile gegenüber den uns jetzt vielfach zur Verfügung stehenden elektronischen Volltextkorpora bietet.

\section{Einige Aspekte der Struktur des Wörterbuchs}

Wörterbücher, deren Einträge sich auf Mehrwortlemmata beziehen, stellen die Bearbeiter häufig vor Lemmatisierungsprobleme. Ein Beispiel aus dem Bereich der Idiomatik sind verbale Wendungen, die sich zwar meistens in der traditionelle Wörterbuchnennform für Verben, im Infinitiv erfassen lassen (z. B. jmdm. sein Herz ausschütten oder einer Sache Tür und Tor öffnen), sich aber in Fällen wie jmdm. blutet das Herz oder jmdm. stehen alle Türen offen genau dieser Zurichtung entziehen. Dass auch bei den so genannten festen Wendungen die Festigkeit in Einzelfällen im aktuellen Sprachgebrauch erheblich variieren kann - ich habe das vor sechzehn Jahren in einem Vortrag auf der damaligen IDS-Tagung am Beispiel hier weht jetzt ein anderer Wind deutlich zu machen versucht -, das erleichtert die lexikografische Arbeit auch nicht gerade.

Erfahrungsgemäß sind in den meisten Fällen alphabetisch sortierte Wörterbücher leichter zu benutzen als thematisch geordnete, zumindest scheinen sie beim breiteren Publikum deutlich beliebter zu sein. Jedenfalls hat Wolf Friederich bei der zweiten Auflage seines idiomatischen Wörterbuchs (1976) die „systematische Anordnung nach Sachgebieten“ der ersten Auflage aufgegeben und das gesamte Werk auf eine alphabetische Sortierung umgestellt. Nun ist aber eine Alphabetisierung strikt nach der Zeichenfolge der Lemmaform auch nicht unbedingt sinnvoll, denn dann müsste jmdm. blutet das Herz unter dem Buchstaben $\mathbf{J}$ eingeordnet werden. Folglich entscheidet sich der 
Lexikograf in der Regel für eines der das Lemma bildenden Wörter, das seiner Einschätzung nach am ehesten vom Benutzer des Wörterbuchs als erfolgversprechender Ausgangspunkt des Nachschlagens angesehen wird. Hier spricht man häufig vom ersten so genannten „sinntragenden“ Wort, obwohl in einer idiomatischen Wendung der Sinn des Einzelwortes ja sehr oft mehr oder weniger stark zugunsten eines Gesamtsinnes der Wendung gelöscht ist. Genauer wäre also von Wörtern zu sprechen, die außerhalb der Wendung als Autosemantika gelten. Das funktioniert in der Praxis recht gut, besonders wenn ein ergiebiges Verweissystem das Problem abfedert, dass Wendungen im täglichen Sprachgebrauch in unterschiedlichen Formen auftreten können: Man findet beispielsweise sowohl das Herz blutete mir (hier würde man unter Herz nachschauen) als auch mir blutete das Herz (hier würde man eher unter bluten nachschauen), folglich sollte im Wörterbuch von bluten auf Herz verwiesen werden, wenn die Wendung unter Herz dargestellt wird, oder umgekehrt.

Wichtig ist, dass im Wörterbuch bei der Benutzung die Sortierung - im Idealfall auch ohne Konsultation der Benutzungshinweise - einsichtig wird; im Duden 11 sollte das dadurch erleichtert werden, dass die Sortierwörter der Wendungen wie eine Art Hauptstichwörter angesetzt und typografisch hervorgehoben sind und auch für die Kolumnentitel am oberen Seitenrand verwendet werden.

Die Mikrostruktur der Einträge im Duden 11 besteht aus sieben Elementen (Artikelpositionen), deren Anordnung traditionellen Schemata von Bedeutungswörterbüchern entspricht: 1. lemmatisierte Formen der Wendung, 2. Pragmatikangaben, 3. Bedeutungsparaphrasen, 4. Anwendungsbeispiele, 5. Belegzitate, 6. Herkunftserläuterungen, 7. Verweise. Die Positionen 1, 3 und möglichst auch 4 sind bei Einträgen, die keine reinen Verweise sind, generell obligatorisch zu besetzen, Position 2 bei nicht standardsprachlichen, nicht allgemeinsprachlichen und nicht überregional oder nur in Österreich oder der Schweiz gebräuchlichen Wendungen. Bezogen auf die oben genannten Zielgruppen des Wörterbuchs sollten für die Lernenden die Bedeutungsparaphrasen sowohl leicht verständlich als auch möglichst treffend sein (was oft nicht ganz leicht vereinbar ist), es sollten vor allem gebrauchstypische Beispiele und Belege angeführt werden. Für die Sprachliebhaber ist es dagegen interessant, auch einmal einer Variation, vielleicht sogar einer sprachspielerischen Abwandlung einer Wendung zu begegnen, und ihnen müssen auch möglichst viele Herkunftsbeschreibungen geboten werden.

Dass die für Duden 11 gewählte Darstellungsform nur eine von mehreren möglichen ist, soll die in der Abbildung 1 vorgeführte Gegenüberstellung mit einer Alternative zeigen, die wir für ein Wörterbuch der Redewendungen und geflügelten Zitate verwendet haben, und bei der der Fokus weg vom Lern-Wörterbuch hin zu einem reinen Lese-Wörterbuch verlagert wurde. 


\begin{tabular}{|c|c|}
\hline DU & $\begin{array}{l}\text { UDEN - Das große Buch der } \\
\text { itate und Redewendungen }\end{array}$ \\
\hline 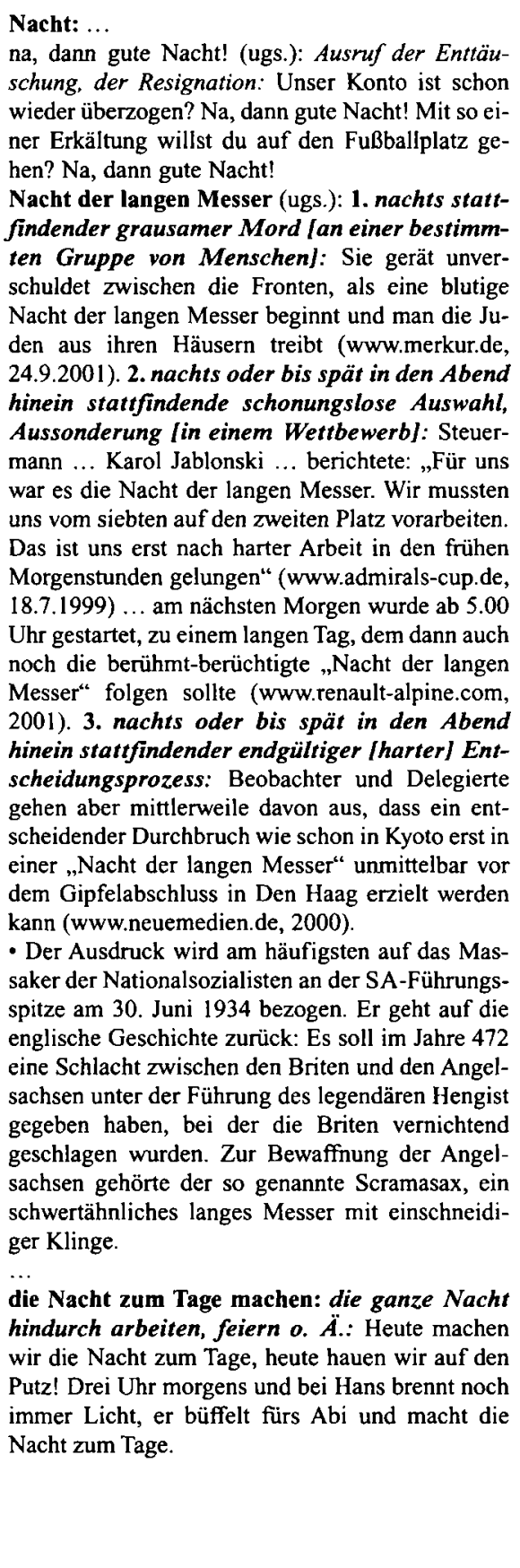 & 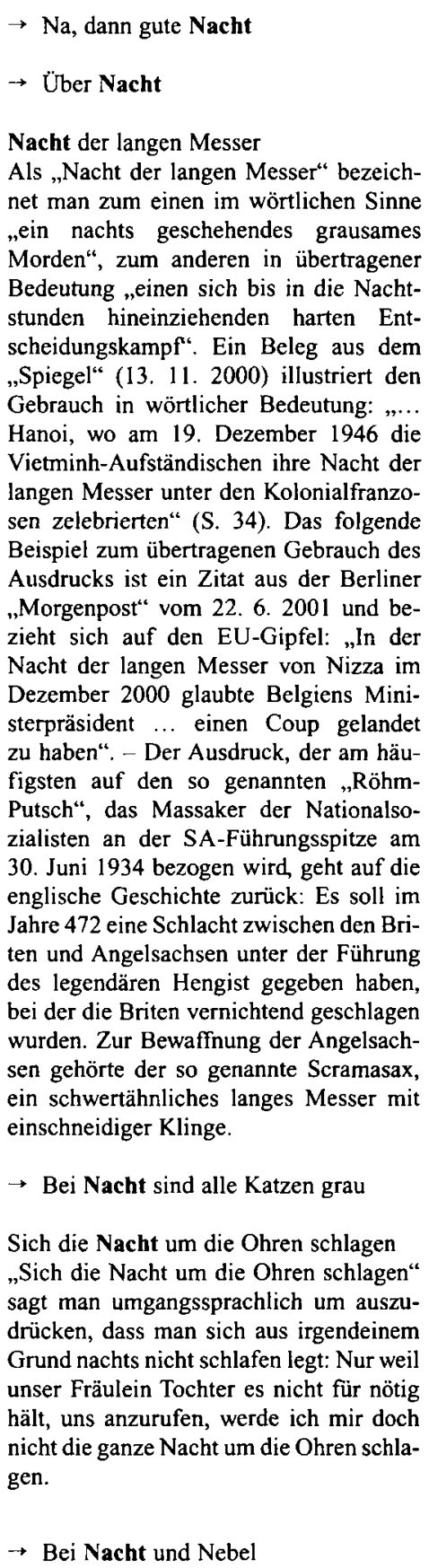 \\
\hline
\end{tabular}


sich (Dativ) die Nacht um die Ohren schlagen (ugs.): die ganze Nacht aufbleiben, nicht zum Schlafen kommen: Die deutschen Fußballfans werden wohl ewig dazu verurteilt bleiben, sich am Fernseher die Nacht um die Ohren zu schlagen (Hörzu 43, 1977, 197).

bei Nacht und Nebel: heimlich [bei Nacht]: ...
Die Nacht zum Tage machen

Wer die Nacht zum Tage macht, legt sich nicht schlafen, sondern durcharbeitet, durchfeiert o. A. die Nacht: Heute machen wir die Nacht zum Tage, ich habe zwölf Flaschen Sekt mitgebracht! - In Friedrich Sieburgs Buch "Robespierre" heißt es: „Aber die Wirte haben es nicht immer leicht, sie müssen Beziehungen haben, die Nacht zum Tage machen und alle verschlungenen Pfade des Schleichhandels kennen“ (S. 168).

Abbildung 1: Strukturelle Alternative zu Duden 11.

\section{Redaktionelle Arbeitstechnik der vorelektronischen Zeit}

Das Manuskript zur ersten Auflage des Duden 11 war ein klassisches handgeschriebenes, bei dem die Schriftauszeichnung für den Druck durch Unterschlängelung und verschiedenfarbige Unterstreichung markiert werden musste. Der handschriftliche Text wurde danach mit der Schreibmaschine abgeschrieben, um die Texterfassung in der Setzerei schneller und fehlerfreier zu machen.

Die Nachteile dieser papiergebundenen Arbeitsweise sind den Älteren unter uns noch sehr vertraut: Textüberarbeitungen, vor allem Umstellungen und spätere Einfügungen sind umständlich und verschlechtern häufig die Erfassbarkeit der Satzvorlage. Gerade bei einem Wörterbuch, dessen Quellen nicht unbedingt in der Weise sortiert sind, dass sie in der Reihenfolge der Wörterbucheinträge vorliegen, können während der Bearbeitung späterer Alphabetabschnitte manche Materialien auftauchen, die für bereits früher formulierte Arbeitsstrecken wichtig sind. (Zum Beispiel könnte sich ein schöner Beleg für die Wendung sich aus der Affäre ziehen, die bereits unter Affäre abgehandelt wurde, erst unter den Karteibelegen für das Wort ziehen finden.) Weiterhin ist bei einem umfassenden Verweissystem die heute mögliche automatische oder wenigstens automatisierte elektronische Verweiskontrolle der damals zu führenden Verweiszettelkartei spürbar überlegen.

Dass unter anderem auch diese heute überholte Arbeitstechnik zu gewissen Inkonsistenzen in der Substanz des Duden 11 geführt hat, ist nicht zu bestreiten. Deren systematischer Abbau ist im Nachhinein so aufwendig, dass auch bei der Bearbeitung der zweiten Auflage trotz der weiterentwickelten technischen Möglichkeiten nicht alle vielleicht wünschenswerten Nachbesserungen verwirklicht werden konnten.

\section{Die Akzeptanz der ersten Auflage}

Das als Dudenband 11 im Jahre 1992 veröffentlichte Wörterbuch der deutschen idiomatischen Wendungen wurde vom Publikum immerhin so wohlwollend aufgenommen und in so befriedigenden Stückzahlen gekauft, dass 
es bis heute seinen Platz in der Duden-Standardreihe bewahrt hat und nach einer rein orthographisch umgestalteten Neuausgabe im Jahre 1997 nun auch in einer inhaltlich überarbeiteten zweiten Auflage seit Januar 2002 vorliegt. Die journalistischen Besprechungen, die über das Abdrucken unseres Pressetextes hinausgingen, waren insgesamt positiv; die linguistische Fachkritik fand ebenso wie einzelne Benutzer des Wörterbuchs einiges auszusetzen, aber einen Totalverriss hat es meines Wissens nicht gegeben. Es wurden im Wesentlichen mangelnde Vollständigkeit einerseits, aber auch zu starke Berücksichtigung von als zu wenig gebräuchlich empfundenen Wendungen gerügt, man bemängelte fehlende Konsistenz der Einträge innerhalb des $\mathrm{Bu}$ ches und im Vergleich zu anderen Wörterbüchern des Dudenverlags, und man äußerte sich kritisch zu pragmatischen Markierungen (nicht zuletzt auch über die regionalen Zuordnungen) und zu nicht hinreichend differenzierenden Bedeutungsangaben sowie zu den verschiedenen Aspekten der Lemmatisierung und der Artikelstruktur.

\section{Vorüberlegungen und Vorarbeiten für die Neuauflage 2002}

Angesichts der insgesamt erfreulichen Akzeptanz, die die erste Auflage gefunden hatte, gab es keinen verlegerischen oder redaktionellen Grund, die Kernkonzeption für eine Neuauflage zu ändern. Ein Teil der projektbezogenen Investitionsmittel wurde für den Import der Satzdaten des Buches in das elektronische Redaktionssystem der Dudenredaktion aufgewendet, wodurch die redaktionelle Bearbeitung zwar erheblich erleichtert wurde, sich aber dafür in einem eher engen finanziellen Rahmen bewegen musste.

Ein Gutachten zur ersten Auflage, das Dimitri Dobrovol'skij freundlicherweise für die Dudenredaktion erstellt hatte, fasste die oben angesprochenen externen Kritikpunkte weitestgehend zusammen und erweiterte sie um eigene Analysen und Vorschläge. Es wurde redaktionell daraufhin überprüft, welche Anregungen innerhalb der Kostenkalkulation mit einiger Aussicht auf Erfolg umsetzbar erschienen. Daraus und aus von der Kritik eher unabhängigen Kriterien ergaben sich die folgenden Bearbeitungsschwerpunkte:

Erstens bestand zehn Jahre nach der Erarbeitung der ersten Auflage ein spürbarer Aktualisierungsbedarf. Die Aufnahme neuer, im Sprachgebrauch erst in den letzten Jahren geläufig gewordener Wendungen (z. B. in trockenen Tüchern sein, die Kuh vom Eis kriegen) ist die für das allgemeine Publikum und damit für die mit dem Vertrieb des Wörterbuchs befassten Abteilungen des Verlags sowie für den Buchhandel die mit Abstand plausibelste Begründung für eine Neuauflage.

Zweitens sollten die Einträge des Duden 11 auf ihre Übereinstimmung mit entsprechenden Einträgen im Großen Wörterbuch der deutschen Sprache, 3. Auflage 1999, geprüft und gegebenenfalls angepasst werden. Gegebenen- 
falls heißt hier: Nur in den Fällen, in denen aus Sicht des Duden 11 und damit in Übereinstimmung mit seiner Konzeption und seiner Zielgruppenorientierung das Große Wörterbuch eine bessere Darstellung bietet, wurde sie übernommen. Rein schematische Konsistenz war nicht erwünscht.

Drittens wurde eine Erweiterung um österreichische und schweizerische Wendungen angestrebt, wie sie in den beiden Duden-Taschenbüchern Wie sagt man in Österreich? von Jakob Ebner und Wie sagt man in der Schweiz? von Kurt Meyer inzwischen vorgegeben waren. (Als die erste Auflage konzipiert wurde, lag das Helvetismen-Taschenbuch noch nicht vor; nun standen beide Werke als Materialbasis zur Verfügung und haben den Duden 11 um so schöne Wendungen wie jmdm. das Goderl kratzen oder auch jmdm. das Hackerl ins Kreuz hauen aus Österreich und jmdm. einen Schlötterling anhängen oder ein Extrazüglein fahren aus der Schweiz bereichert.)

Viertens sollten die Quellenbelege teilweise durch jüngere ausgetauscht und um neue Belegzitate, auch aus Internettexten, erweitert werden, um die Authentizität und die Aktualität der Darstellung noch deutlicher werden zu lassen.

Schließlich hatten wir uns einige kleinere formale Änderungen vorgenommen, die zu einer Verbesserung der „Expertennutzung“ des Wörterbuchs führen könnten: Dazu gehört die Unterscheidung zwischen dem Platzhalter etwas für nicht personelle substantivische Ergänzungen, den wir jetzt in der abgekürzten Form etw. ansetzen, und dem unveränderlichen Indefinitpronomen etwas, das weiterhin unabgekürzt bleibt, in der Lemmaform der Wendungen sowie in den Bedeutungsparaphrasen (sich etw. unter den Nagel reißen vs. jmdm. etwas husten). Dazu gehört auch die optisch klarere Abgrenzung zwischen normierten Beispielen aus der Feder der Wörterbuchautoren und den wörtlichen Belegzitaten aus Korpora durch einen so genannten Mittepunkt. Außerdem sollte das Reflexivum sich in den Fällen, in denen es in der Lemmaform keinen Akkusativ repäsentiert, durch den Hinweis $<$ Dativ $>$ markiert werden.

Unabhängig von den inhaltlichen und formalen redaktionellen Überarbeitungspunkten zeigt sich die zweite Auflage des Duden 11 auch in Typographie, Farbigkeit, Buchformat und Seitenlayout verändert. Hier fand eine Anpassung an die mit der 22. Auflage der Duden-Rechtschreibung modernisierte Reihengestaltung der heute zwölfbändigen Standard-Dudenreihe statt. Um die optische Attraktivität des Wörterbuchs für das allgemeine Publikum noch zusätzlich zu erhöhen, wurden einige ganzseitige Illustrationen eingefügt.

\section{Die Ausführung der Neubearbeitung}

Die wie oben dargelegt konzipierte Bearbeitung konnte zu etwa 80 Prozent von einer externen Redakteurin auf einer Papierfahne durchgeführt werden. Allerdings musste ihre Arbeit aus verschiedenen redaktionsinternen Quellen und Materialien gespeist werden. Hierzu wurde vor allem die elektronische 
Duden-Sprachkartei abgefragt, die im Unterschied zur älteren Kartensammlung einen gezielten Zugriff auf die exzerpierten Wendungen erlaubt (vgl. Abbildung 2).

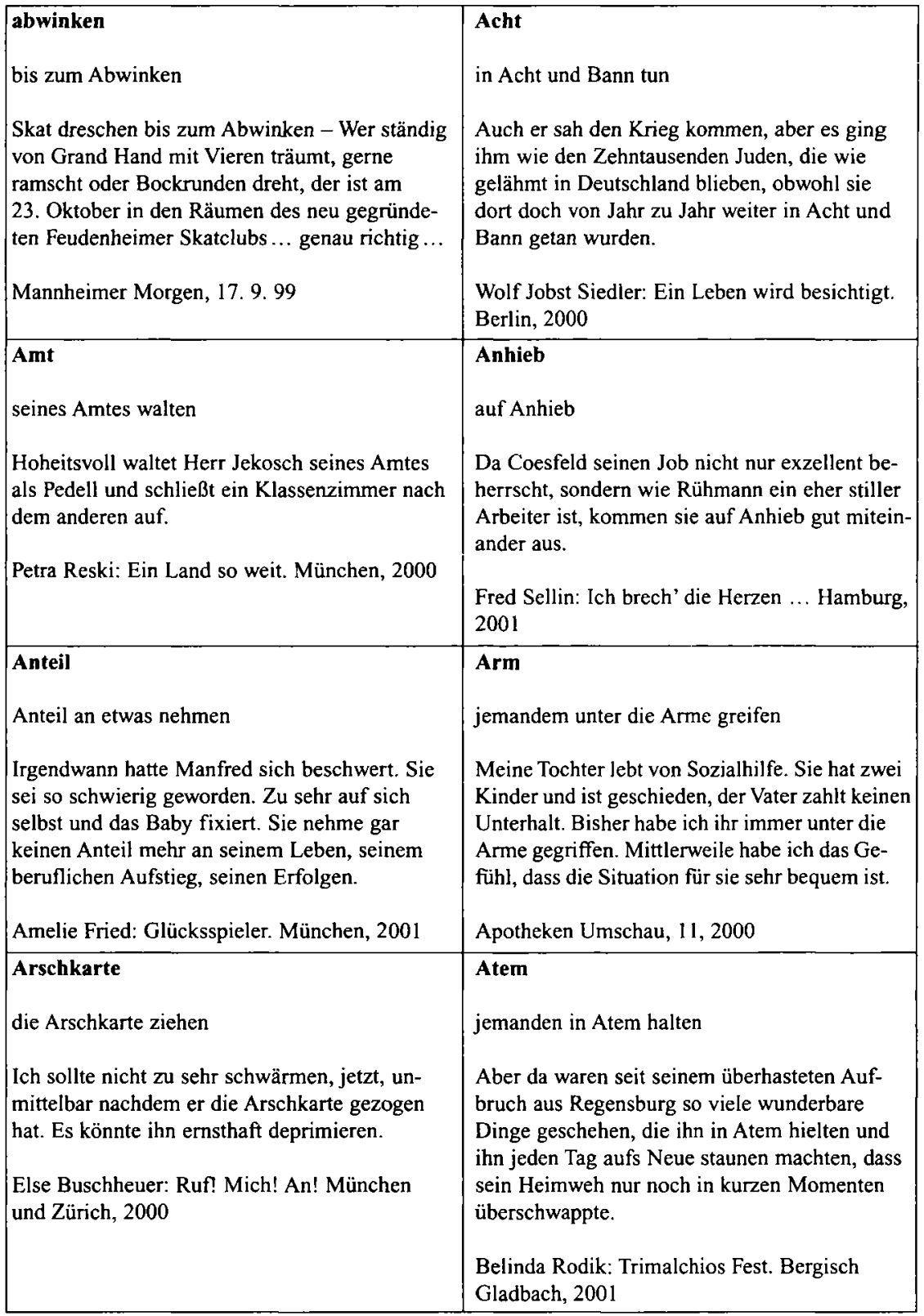

Abbildung 2: Belegbeispiele aus der elektronischen Dudenkartei. 
Weiterhin konnten der Bearbeiterin ergänzende Hinweise aus einer speziell für Duden 11 geführten Nachtrags- und Korrekturkartei zur Verfügung gestellt werden. Wo die externe Redakteurin allein nicht weiterkam, konnte sie auf die Unterstützung der verlagsinternen Redaktion zurückgreifen.

Überwiegend redaktionsintern bearbeitet wurden aus Termingründen etwa 20 Prozent des Wörterbuchtextes und alle Stellen des Gesamttextes, bei denen ein kritisches Gegenlesen einen Änderungs- oder Ergänzungsbedarf gegenüber der ersten externen Bearbeitungsphase aufzeigte. Im bei diesem Projekt nur redaktionsintern nutzbaren elektronischen Datenbestand wurden besonders auch solche Operationen durchgeführt, die mit einer Volltextsuche erheblich erleichtert werden konnten (z. B. die banale, aber durchaus nötige inhaltliche Aktualisierung von normierten Beispielen durch das Ersetzen von Mark durch Euro).

\section{Ausblick auf eine zukünftige Neubearbeitung: Die allgemeinen Rahmenbedingungen}

Das nun Folgende ist, darauf ist ausdrücklich und nachdrücklich hinzuweisen, in hohem Maße spekulativ. Niemand weiß heute, zum Zeitpunkt der IDS-Tagung 2003, wann eine Neuauflage des Duden 11 zu planen und auszuführen sein wird, und ebensowenig lässt sich heute schon im Vorhinein festlegen, welcher Art die Veränderungen sein und welchen Umfang sie haben werden. Denn das hängt von den verschiedensten Faktoren ab, deren Entwicklung kaum präzise vorauszusagen ist. Dazu gehören unter anderem der wirtschaftliche Erfolg der zweiten Auflage, also der Absatz des Buches; weiterhin die Wettbewerbssituation auf dem Buchmarkt oder im Online- und Offline-Bereich; außerdem die werkübergreifende strategische Planung innerhalb des Dudenverlags, die künftig zur Verfügung stehenden personellen internen und externen Kapazitäten und Investitionsmittel für das Gesamtprogramm, und auch - und sicher nicht ganz zuletzt - die Rückmeldungen über die Brauchbarkeit des Buches von den allgemeinen Benutzern und den linguistischen und nicht linguistischen Wörterbuchkritikern sowie die Forschungssituation in der wissenschaftlichen lexikografischen und lexikologischen Arbeit im Bereich der idiomatischen Wendungen.

\section{Noch einmal das Thema Aktualität}

Trotz all dieser Unwägbarkeiten sind bestimmte Vermutungen hinsichtlich einer Neubearbeitung nicht ohne Plausibilität. Mit einiger Sicherheit wird die Aktualisierung immer ein Schwerpunkt sein: Nicht nur neue Wendungen werden entstehen (in unserer „Warteschleife“ gibt es derzeit z. B. ein großes Rad drehen oder Geld/einen Betrag in die Hand nehmen), es werden auch einige weniger gebräuchlich werden. (Vielleicht ist das heute schon als veraltend markierte $j m d m$. Kattun geben ein so genannter ,Streichkandidat“ bei einer Neuauflage.) Einige werden sich in ihrer Form modifizieren (man den- 
ke an die jüngeren umgangssprachlichen und saloppen Varianten zu jmdm. auf die Nerven gehen wie etwa jmdm. auf den Senkel gehen oder jmdm. auf den Zeiger gehen), bei einigen wird sich die Verwendungsweise ändern, so dass neue Bedeutungsparaphrasen zu erarbeiten sein werden, und es könnte bei der einen oder anderen Wendung eine Deidiomatisierung stattfinden all das und sicher noch einiges andere würde vielleicht schon heute und in den kommenden Jahren sicher zunehmend Anlass bieten, die vorliegende Auflage kritisch durchzusehen und an mehreren Stellen zu überarbeiten.

Wird an dem Prinzip festgehalten, die normierten, von Lexikografen formulierten Anwendungsbeispiele durch wörtlich zitierte Quellenbelege zumindest zu ergänzen, ist auch hier ein Aktualisierungsansatz. Außerdem kann angesichts der wahrscheinlich noch weiter zunehmenden Zugänglichkeit von elektronischen Korpora für redaktionelle Wörterbucharbeit diskutiert werden, ob und in welchem Maße der Zitierbereich ausgeweitet werden sollte, ob möglicherweise in bestimmten Fällen verstärkt das normierte Beispiel vollständig durch den Beleg zu ersetzen wäre.

\section{Und nicht zu vergessen: die Erträge von Kritik und Forschung}

Aus der prinzipiellen Problematik der optimalen Lemmatisierung von Wendungen und ihrer Anordnung im Wörterbuch könnten sich aus der lexikografisch-lexikologischen Forschung zu den bereits vorhandenen Ansätzen noch weitere Erkenntnisse für eine Neuorientierung im Duden 11 ergeben; auch in der Mikrostruktur ist die optimale Struktur heute vielleicht noch nicht verwirklicht. Der hohe redaktionelle Aufwand allerdings, der - soweit ich das zurzeit einschätzen kann - mit Verbesserungen in diesen Bereichen verbunden sein würde, setzt voraus, dass die allgemeine Benutzbarkeit des Buches dadurch tatsächlich bemerkbar optimiert wird, was nicht nur schwer zu erreichen sondern auch schwer zu beweisen sein dürfte, besser gesagt: was den für das Marketing, die Pressearbeit und den Vertrieb zuständigen Abteilungen des Verlages und auch der Redaktion nicht immer hinreichend augenfällig wird. Im Bereich der strukturellen Fortentwicklung des Wörterbuchs werden also aller Wahrscheinlichkeit nach zunächst nur kleine Schritte realisierbar sein.

Denkbar ist angesichts der derzeitigen Anstrengungen, die elektronischen Volltextkorpora auch im Hinblick auf Kollokationen und damit in gewisser Weise auch auf idiomatischen Wendungen besser nutzbar zu machen, dass künftige Bearbeitungen des Duden 11 verstärkt auf maschinell unterstützten Korpusabgleich setzen. Da aber die semantische Blindheit der Korpusaufbereitung auf absehbare Zeit im Wesentlichen erhalten bleiben dürfte, werden nach meiner Einschätzung die von Menschen erstellten Exzerptionssammlungen keineswegs überflüssig. Eine sehr nützliche Ergänzung und Validierung durch verbesserte Korpusnutzung ist dagegen durchaus auch in 
der näheren Zukunft schon zu erwarten. Möglicherweise wird das einer der stärkeren Impulse aus der wissenschaftlichen Befassung mit der Lexikografie der Wendungen sein, der sich unmittelbar auf die Gestaltung und die Inhalte eines Wörterbuchs wie des Duden 11 auswirkt.

\section{Literatur}

Der Große Duden - Bedeutungswörterbuch (1970): 1. Auflage. Mannheim/Wien/Zürich. Der Große Duden - Stilwörterbuch (1934): 1. Auflage. Leipzig.

Duden - Das große Wörterbuch der deutschen Sprache in sechs Bänden (1976-1981): 1. Auflage. Mannheim/Wien/Zürich.

Duden - Das große Wörterbuch der deutschen Sprache in zehn Bänden (1999): 3. Auflage. Mannheim/Leipzig/Wien/Zürich.

Duden - Das Stilwörterbuch (1971): 6. Auflage. Mannheim/Wien/Zürich.

Duden - Redewendungen und sprichwörtliche Redensarten. Wörterbuch der deutschen Idiomatik (1992): 1. Auflage. Mannheim/Leipzig/Wien/Zürich.

Duden - Redewendungen. Wörterbuch der deutschen Idiomatik (2002): 2. Auflage. Mannheim/Leipzig/Wien/Zürich.

Ebner, Jakob (1998): Duden - Wie sagt man in Österreich? Wörterbuch des österreichischen Deutsch. 3. Auflage. Mannheim/Leipzig/Wien/Zürich.

Friederich, Wolf (1966): Moderne deutsche Idiomatik. Systematisches Wörterbuch mit Definitionen und Beispielen. 1. Auflage. München.

Friederich, Wolf (1976): Moderne deutsche Idiomatik. Alphabetisches Wörterbuch mit Definitionen und Beispielen. 2. Auflage. München.

Krüger-Lorenzen, Kurt (1960): Das geht auf keine Kuhhaut. Deutsche Redensarten - und was dahinter steckt. 1. Auflage. Düsseldorf

Meyer, Kurt (1989): Duden - Wie sagt man in der Schweiz? Wörterbuch der schweizerischen Besonderheiten. 1. Auflage. Mannheim/Wien/Zürich.

Scholze-Stubenrecht, Werner (1988): Phraseologismen im Wörterbuch. In: Harras, Gisela (Hg.): Das Wörterbuch. Artikel und Verweisstrukturen. Jahrbuch 1987 des Instituts für deutsche Sprache (Sprache der Gegenwart 74). S. 284-302. 